\title{
A construction of noncontractible simply connected cell-like two-dimensional Peano continua
}

\author{
by \\ Katsuya Eda (Tokyo), Umed H. Karimov (Dushanbe) and \\ Dušan Repovš (Ljubljana)
}

\begin{abstract}
Using the topologist sine curve we present a new functorial construction of cone-like spaces, starting in the category of all path-connected topological spaces with a base point and continuous maps, and ending in the subcategory of all simply connected spaces. If one starts from a noncontractible $n$-dimensional Peano continuum for any $n>0$, then our construction yields a simply connected noncontractible $(n+1)$-dimensional celllike Peano continuum. In particular, starting from the circle $\mathbb{S}^{1}$, one gets a noncontractible simply connected cell-like 2-dimensional Peano continuum.
\end{abstract}

1. Introduction. It is well known that all cell-like polyhedra are contractible. Griffiths [5] constructed a 2-dimensional nonsimply connected celllike Peano continuum: Let $\mathbb{H}_{1}$ be the 1-dimensional Hawaiian earrings with the base point $\theta$ at which $\mathbb{H}_{1}$ is not locally simply connected. Let $Y=C\left(\mathbb{H}_{1}\right)$ be the cone over $\mathbb{H}_{1}$. Then $\mathbb{H}_{1}$ can be considered as the base of the cone $C\left(\mathbb{H}_{1}\right)$ and $\theta$ as its base point. The Griffiths space is then defined as the bouquet of two copies of $Y$ with respect to the point $\theta$.

A generalization of the Griffiths example is analogous - instead of the 1-dimensional one considers the 2-dimensional Hawaiian earrings [3], i.e. the subspace $\mathbb{H}_{2}$ of the 3 -dimensional Euclidean space, $\mathbb{H}_{2}=\left\{\left(x_{0}, x_{1}, x_{2}\right) \in\right.$ $\left.\mathbb{R}^{3} \mid\left(x_{0}-1 / k\right)^{2}+x_{1}^{2}+x_{2}^{2}=(1 / k)^{2}, k \in \mathbb{N}\right\}$. It is easy to see that this generalization of the Griffiths example is a 3 -dimensional noncontractible simply connected cell-like Peano continuum.

The purpose of the present paper is to construct a functor $S C(-,-)$ from the category of all path-connected spaces with a base point and continuous

2000 Mathematics Subject Classification: Primary 54F15, 54G15, 57N60; Secondary 54C55, 55M15, 55Q52.

Key words and phrases: Peano continuum, acyclicity, cell-like set, noncontractible compactum, cone-like space. 
mappings, to the subcategory of all simply connected spaces with a base point. The following are our main results:

THEOREM 1.1. For every path-connected space $Z$ with $z_{0} \in Z$, the space $S C\left(Z, z_{0}\right)$ is simply connected.

THEOREM 1.2. For every noncontractible space $Z$ with $z_{0} \in Z$, the space $S C\left(Z, z_{0}\right)$ is noncontractible.

If $Z$ is a Peano continuum, then $S C\left(Z, z_{0}\right)$ is also a Peano continuum. If $Z$ is an $n$-dimensional metrizable space for $n>0$, then the space $S C\left(Z, z_{0}\right)$ is $(n+1)$-dimensional. If $Z$ is compact, then $S C\left(Z, z_{0}\right)$ is a compact space with trivial shape.

In particular, when $Z$ is the circle $\mathbb{S}^{1}$, we get the following:

Corollary 1.3. For any point $z_{0}$ of the circle $\mathbb{S}^{1}$, the space $S C\left(\mathbb{S}^{1}, z_{0}\right)$ is a noncontractible simply connected cell-like 2-dimensional Peano continuum.

As a general reference for algebraic topology we refer the reader to [10].

2. Preliminaries. For any two points $A$ and $B$ in the plane $\mathbb{R}^{2},[A, B]$ denotes the linear segment connecting these points. For $a, b \in \mathbb{R}$ with $a<b$, $[a, b]$ denotes the closed interval, $(a, b)$ denotes the open interval and $[a, b)$ and $(a, b]$ denote the half-open intervals, as usual. The unit interval $[0,1]$ will be denoted by $\mathbb{I}$. To avoid confusion between an open interval and an element of the square $\mathbb{I} \times \mathbb{I}$, we shall write $(a ; b)$ for the latter, where $a, b \in \mathbb{I}$. Our construction is based on the piecewise linear topologist sine curve $T$ in the plane. Let $A_{n}=(1 / n ; 0), B_{n}=(1 / n ; 1)$, for $n \in \mathbb{N}=\{1,2,3, \ldots\}$, $A=(0 ; 0), B=(0 ; 1)$ be the points of the plane $\mathbb{R}^{2}$. Let $L_{2 n-1}=\left[A_{n}, B_{n}\right]$ and $L_{2 n}=\left[B_{n}, A_{n+1}\right]$. The space $T$ is the subspace of $\mathbb{I}^{2}$ defined as the union of all segments $L_{n}$ and $L=[A, B]$.

Let $Z$ be any space with a base point $z_{0}$. Then the base set of $S C\left(Z, z_{0}\right)$ is the quotient set of $T \times Z \cup \mathbb{I}^{2}$ obtained by the identification of the points $\left(s, z_{0}\right) \in T \times Z$ with $s \in T \subset \mathbb{I}^{2}$ and by the identification of each set $\{s\} \times Z$ with the one-point set $\{s\}$ if $s \in L$. There is a natural projection $p: S C\left(Z, z_{0}\right) \rightarrow \mathbb{I}^{2}$. To $p$ there corresponds a pair of functions $p_{1}$ and $p_{2}$ such that $p(z)=\left(p_{1}(z) ; p_{2}(z)\right)$. For $a=(x ; y) \in T$ with $x>0$, the set $p^{-1}(a)$ is denoted by $Z_{a}$, which is homeomorphic to $Z$, and for $y \in \mathbb{I}$ the set $p_{2}^{-1}(\{y\})$ is denoted by $M_{y}$. Let $O_{\varepsilon}(a)=p^{-1}\left(U_{\varepsilon}(a)\right)$, where $U_{\varepsilon}(a)$ is the open $\varepsilon$-ball with center at $a \in \mathbb{I} \times \mathbb{I}$ with respect to the standard metric.

The topology of $S C\left(Z, z_{0}\right)$ coincides with the quotient topology at each point outside $L$. A basic neighborhood of a point $a=(0 ; y) \in L$ is of the form $O_{\varepsilon}(a)$. Therefore, the topology of $S C\left(Z, z_{0}\right)$ is the quotient topology when $Z$ is compact. 
Obviously, $S C(-,-)$ is a functor from the category of topological spaces with a base point to itself. The space $S C\left(Z, z_{0}\right)$ is path-connected, pathconnected and locally connected, finite-dimensional, metrizable or compact if $Z$ is path-connected, path-connected and locally connected, metrizable or compact, respectively. In particular, $S C\left(Z, z_{0}\right)$ is a Peano continuum if $Z$ is a Peano continuum.

If $Z$ is compact, $S C(Z)$ is a quotient space of $T \times Z \cup \mathbb{I}^{2}$ and hence $S C(Z)$ is also compact. Next we show that the shape type of $S C(Z)$ is that of the one-point space when $Z$ is compact. To see this let $\mathcal{U}$ be an open cover of $S C(Z)$. By the compactness of $\mathbb{I}$ we have $\varepsilon>0$ such that $p^{-1}([0, \varepsilon) \times[a, b])$ is contained in an element of $\mathcal{U}$ for every $0 \leq a<b \leq 1$ with $b-a<\varepsilon$. By the compactness of $Z$ we also have a cover $O_{1}, \ldots, O_{m}$ of $X$ and points $P_{1}=A_{1}, \ldots, P_{n}$ on $T$ such that $\left[P_{i}, P_{i+1}\right] \subset T \cap[\varepsilon, 1] \times \mathbb{I}$ and each $\left[P_{i}, P_{i+1}\right] \times O_{j}$ is contained in an element of $\mathcal{U}$. Hence we have a refinement of $\mathcal{U}$ whose nerve is contractible. This yields the conclusion.

Let $Z$ be an $n$-dimensional metrizable space for $n>0$. Then, since $S C(Z)=\mathbb{I}^{2} \cup \bigcup_{n=1}^{\infty} p^{-1}([1 / n, 1] \times \mathbb{I})$ and $p^{-1}([1 / n, 1] \times \mathbb{I} \cap T)$ is homeomorphic to $\mathbb{I} \times Z$, the dimension of $S C(Z)$ is $n+1$ by [4, Theorems 4.1 .3 and 4.1.9] and [9, p. 221]. Hence $S C(Z)$ is a cell-like, $(n+1)$-dimensional compact metrizable space if $Z$ is an $n$-dimensional compact metrizable space $[7,8]$.

A path in $X$ is a continuous mapping of the closed interval $[a, b]$ to $X$. We say that two paths are homotopic if they are defined on the same domain and are homotopic relative to their ends. The composition of two paths $f$ : $[a, b] \rightarrow X$ and $g:[b, c] \rightarrow X$ such that $f(b)=g(b)$ is a path $h:[a, c] \rightarrow X$ which is defined as follows:

$$
h(t)= \begin{cases}f(t) & \text { if } a \leq t \leq b \\ g(t) & \text { if } b \leq t \leq c\end{cases}
$$

Let $f:[a, b] \rightarrow X$ and $g:[c, d] \rightarrow X$ be paths. We say that $f$ is equivalent to $g$ and write $f \cong g$ when $f(a+(b-a) t)=g(c+(d-c) t)$ for each $t \in \mathbb{I}$ and define $\bar{f}$ as $\bar{f}(t)=f(a+b-t)$ for each $a \leq t \leq b$.

A loop with base point $x_{0}$ in a space $X$ is a path $f:[a, b] \rightarrow X$ for which $f(a)=f(b)=x_{0}$.

The product of two loops is defined in the standard way. The constant mapping to $\left\{x_{0}\right\}$ is denoted by $c_{x_{0}}$. Let $f:[a, b] \rightarrow X$ be a path, $c$ any point in $[a, b]$ and $\alpha$ any loop with base point $f(c)$. The modification of the path along a loop $\alpha: \mathbb{I} \rightarrow X$ is the path $g:[a, b] \rightarrow X$ which is defined for an interval $\left[t_{1}, t_{2}\right] \subset(a, b)$ such that $c \in\left[t_{1}, t_{2}\right]$ as follows:

$$
g(s)= \begin{cases}f\left((s-a)(c-a) /\left(t_{1}-a\right)+a\right) & \text { if } a \leq s \leq t_{1} \\ \alpha\left(\left(s-t_{1}\right) /\left(t_{2}-t_{1}\right)\right) & \text { if } t_{1} \leq s \leq t_{2} \\ f\left((b-s)(b-c) /\left(b-t_{2}\right)+b\right) & \text { if } t_{2} \leq s \leq b\end{cases}
$$


The definition of the modification of paths depends on the interval $\left[t_{1}, t_{2}\right]$, but all such paths are homotopic. (For simplicity of the definition we suppose that the domain of a loop $\alpha$ is $\mathbb{I}$, but later we shall use a variant of the modification for loops with arbitrary domains.)

A homotopy connecting an injective mapping with a constant one is called a contraction. Whenever possible we shall use the symbol $S C(Z)$ instead of $S C\left(Z, z_{0}\right)$.

\section{Proof of Theorem 1.1}

Lemma 3.1. Let $A$ be a strong deformation retract of $X$ and let $\alpha$ : $[0,1] \rightarrow X$ be a path with end points $\alpha(0)$ and $\alpha(1)$ in $A$. Then there exists a path $\alpha^{\prime}:[0,1] \rightarrow A \subset X$ which is homotopic to $\alpha$.

Proof. The assertion follows directly from the definition of the strong deformation retraction.

Lemma 3.2. Let $X$ be any space and $\alpha=\left(\alpha_{1}, \alpha_{2}\right)$ any path in $X \times \mathbb{I}$ with end points $\alpha(0) \in X \times\{0\}$ and $\alpha(1) \in X \times\{1\}$. Then there exists a path $\alpha^{\prime}$ in $X \times \mathbb{I}$ homotopic to $\alpha$ and such that $\operatorname{Im}\left(\alpha^{\prime}\right) \subset\left\{\alpha_{1}(0)\right\} \times \mathbb{I} \cup X \times\{1\}$.

Proof. Let $H: \mathbb{I} \times \mathbb{I} \rightarrow X \times \mathbb{I}$ be the homotopy defined by

$$
H(s, t)= \begin{cases}\left(\alpha_{1}(0), 2 s\right) & \text { if } 0 \leq s \leq t / 2, \\ \left(\alpha_{1}\left(\frac{2 s-t}{2-t}\right),(1-t) \alpha_{2}\left(\frac{2 s-t}{2-t}\right)+t\right) & \text { if } t / 2 \leq s \leq 1\end{cases}
$$

Obviously, we have $H(0, t)=\alpha(0), H(1, t)=\alpha(1), H(s, 0)=\alpha(s)$ and $\operatorname{Im}(H(-, 1)) \subset\left\{\alpha_{1}(0)\right\} \times \mathbb{I} \cup X \times\{1\}$ so $H$ is the desired homotopy connecting $\alpha$ and $\alpha^{\prime}=H(-, 1)$.

The path $\left.\alpha^{\prime}\right|_{[0,1 / 2]}$ is called the linear part and $\left.\alpha^{\prime}\right|_{[1 / 2,1]}$ the residual part of $\alpha^{\prime}$.

In the following lemmata we use the symbols $B_{n}, Z_{B_{n}}, U_{\varepsilon}\left(B_{n}\right), O_{\delta}\left(B_{n}\right)$ and $M_{0}$, defined in Section 2.

Lemma 3.3. Let $f: \mathbb{I} \rightarrow S C(Z)$ be any path. Then for every $n \in \mathbb{N}$ and every $\varepsilon>0$ there exist a path $f_{n, \varepsilon}: \mathbb{I} \rightarrow S C(Z)$ and a homotopy $H_{n, \varepsilon}: \mathbb{I}^{2} \rightarrow S C(Z)$ such that:

(1) $H_{n, \varepsilon}(s, 0)=f(s), H_{n, \varepsilon}(s, 1)=f_{n, \varepsilon}(s)$;

(2) $\operatorname{Im}\left(f_{n, \varepsilon}\right) \cap Z_{B_{n}}=\emptyset$;

(3) $H_{n, \varepsilon}(s, t)=f(s)$ if $f(s) \notin O_{\varepsilon}\left(B_{n}\right)$.

Proof. Let $\delta$ be a number such that $0<\delta<\varepsilon$ and $U_{\delta}\left(B_{n}\right) \cap T=$ $U_{\delta}\left(B_{n}\right) \cap\left(L_{2 n-1} \cup L_{2 n}\right)$. Since $f^{-1}\left(Z_{B_{n}}\right)$ is a compact subset of $f^{-1}\left(O_{\delta}\left(B_{n}\right)\right)$, there exists a finite set of pairwise disjoint intervals $\left\{\left[a_{k}, b_{k}\right]: k \in K_{n}\right\}$ which cover $f^{-1}\left(Z_{B_{n}}\right)$ in $f^{-1}\left(O_{\delta}\left(B_{n}\right)\right)$ and whose end points lie outside $f^{-1}\left(Z_{B_{n}}\right)$. 
Using the modifications of paths along loops we can assume without loss of generality that the end points of all paths lie on $T$. For a given $k \in K_{n}$ consider the path $\left.f\right|_{\left[a_{k}, b_{k}\right]}:\left[a_{k}, b_{k}\right] \rightarrow O_{\delta}\left(B_{n}\right)$. Since $p^{-1}\left(U_{\delta}\left(B_{n}\right) \cap T\right)$ is a strong deformation retract of $O_{\delta}\left(B_{n}\right)$, the path $\left.f\right|_{\left[a_{k}, b_{k}\right]}$ is homotopic to a path $f_{n, k}:\left[a_{k}, b_{k}\right] \rightarrow p^{-1}\left(U_{\delta}\left(B_{n}\right) \cap T\right) \subset O_{\delta}\left(B_{n}\right)$ by Lemma 3.1.

The space $p^{-1}\left(U_{\delta}\left(B_{n}\right) \cap T\right)$ is naturally homeomorphic to the product of the interval and the space $Z$. The product $\left(\left[f\left(a_{k}\right), B_{n}\right] \cup\left[B_{n}, f\left(b_{k}\right)\right]\right) \times Z$ is a strong deformation retract of $p^{-1}\left(U_{\delta}\left(B_{n}\right) \cap T\right)$. Therefore the path $f$ : $\left[a_{k}, b_{k}\right] \rightarrow O_{\delta}\left(B_{n}\right)$ is homotopic to a path in $\left(\left[f\left(a_{k}\right), B_{n}\right] \cup\left[B_{n}, f\left(b_{k}\right)\right]\right) \times Z$, again by Lemma 3.1. By Lemma 3.2, the path $f:\left[a_{k}, b_{k}\right] \rightarrow O_{\delta}\left(B_{n}\right)$ is homotopic to a path whose linear part lies in $L_{2 n-1} \cup L_{2 n}$ and whose residual part does not intersect $Z_{B_{n}}$. The linear part can be slightly deformed in $\mathbb{I} \times \mathbb{I}$ to a segment $\left[f\left(a_{k}\right), f\left(b_{k}\right)\right]$ with fixed ends $f\left(a_{k}\right)$ and $f\left(b_{k}\right)$, which does not contain the point $B_{n}$. Since the index $k$ is arbitrary and the number of the intervals $\left\{\left[a_{k}, b_{k}\right]: k \in K_{n}\right\}$ is finite we get the desired mapping $f_{n, \varepsilon}$.

The next lemma is a direct consequence of Lemma 3.3:

Lemma 3.4. Any loop in $S C(Z)$ with base point in $M_{0}$ is homotopic to a loop in $S C(Z) \backslash \bigcup_{n \in \mathbb{N}} Z_{B_{n}}$.

Lemma 3.5. $M_{0}$ is a strong deformation retract of $S C(Z) \backslash \bigcup_{n \in \mathbb{N}} Z_{B_{n}}$.

Proof. The deformation $D:\left(S C(Z) \backslash \bigcup_{n \in \mathbb{N}} Z_{B_{n}}\right) \times \mathbb{I} \rightarrow S C(Z) \backslash$ $\bigcup_{n \in \mathbb{N}} Z_{B_{n}}$ is given by the piecewise linear mapping (linear over every triangle $A_{n} B_{n} A_{n+1}$ and $\left.A_{n+1} B_{n+1} B_{n}\right)$ which maps $\left[A_{n}, B_{n}\right] \backslash\left\{B_{n}\right\}$ and $\left[A_{n+1}, B_{n}\right] \backslash\left\{B_{n}\right\}$ to the points $A_{n}$ and $A_{n+1}$, respectively (see Figure 1 ). Since the spaces $Z_{B_{n}}$ have been deleted, $D$ is well-defined and continuous.

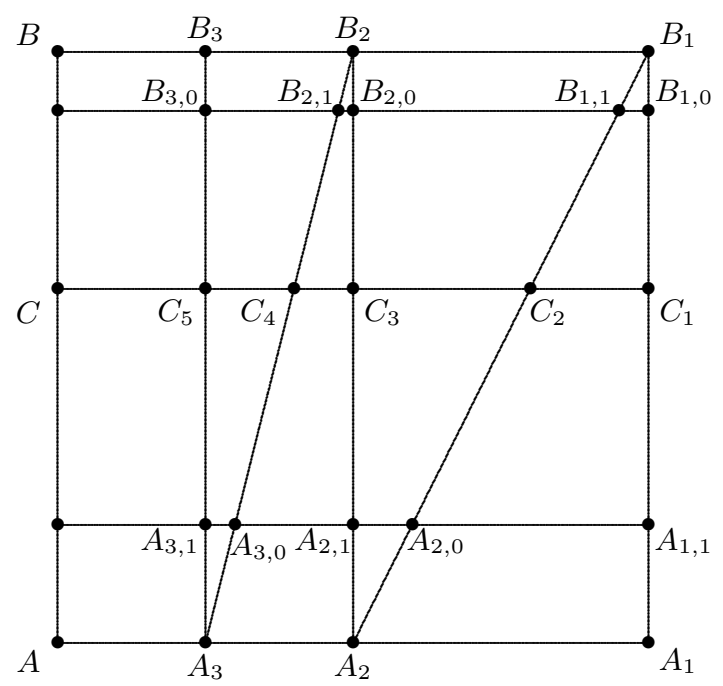

Fig. 1 
The following is a consequence of Lemmata 3.4 and 3.5:

Lemma 3.6. Let $f$ be a loop in $S C(Z)$ with base point in $M_{0}$. Then $f$ is homotopic to a loop in $M_{0}$.

Before we show the simple connectedness of $S C(Z)$, we exhibit a homotopy from the canonical winding to a constant in the case when $Z$ is the circle in Figure 2. In the remaining part of this section we shall use the word "homotopic" for loops in a weaker sense, that is, two loops $f$ and $g$ will be considered to be homotopic if there exists a homotopy $H(-, t)$ such that $H(-, 0)=f, H(-, 1)=g$ and $H(-, t)$ is a loop for each $t$. From a loop like I in Figure 2 we pull the bottom of the loop to the left. This is procedure I. Then we pull up to the loop like III through the one like II; this is procedure II. Now the loop is in the upper edge without tangles, like III. We contract the loop to the point $B$; this is procedure III. To generalize these simple procedures I, II, III, we need to describe them more precisely.

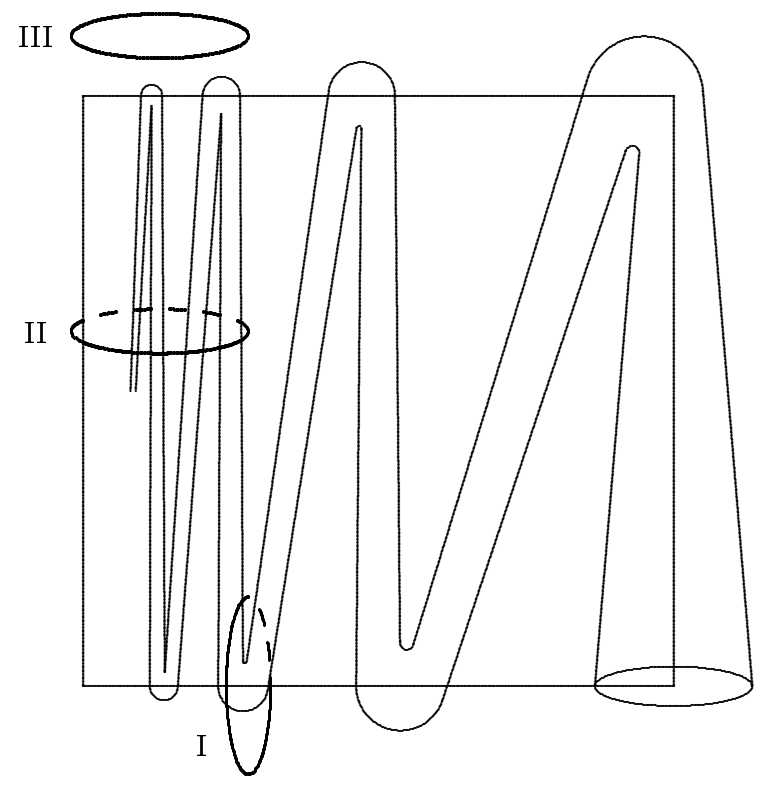

Fig. 2

For a loop $\alpha$ in $Z$ with base point $z_{0}$ and a point $u \in T$, let $\alpha_{u}$ be a loop in $Z_{u}$ induced naturally by the homeomorphism between $Z$ and $Z_{u}$, i.e. $\alpha_{u}(t)=(u, \alpha(t))$; in particular the base point of $\alpha_{u}$ is $u$.

We call $\beta:[a, b] \rightarrow S C(Z)$ a basic loop at $A_{n}$ if there exists a loop $\alpha$ in $Z$ with base point $z_{0}$ such that

(a) $\beta(a)=\beta(b)=A, \beta((2 a+b) / 3)=\beta((a+b) / 2)=A_{n}$;

(b) $\left.\beta\right|_{[a,(2 a+b) / 3]}$ and $\left.\beta\right|_{[(a+b) / 2, b]}$ are linear mappings;

(c) $\left.\beta\right|_{[(2 a+b) / 3,(a+b) / 2]} \cong \alpha_{A_{n}}$. 
LEMma 3.7. Any basic loop $\beta:[0,1] \rightarrow S C(Z)$ at $A_{n}$ is homotopic to the constant mapping $B$ in the subspace $p^{-1}\left(\left[A, A_{n}\right] \times \mathbb{I}\right)$.

Proof. We modify $\beta$ to $\gamma_{0}$ so that:

(1) $\gamma_{0}(0)=\beta(0)=A,\left.\gamma_{0}\right|_{[1 / 3,1]}=\left.\beta\right|_{[1 / 3,1]}$;

(2) $\left.\gamma_{0}\right|_{[1 /(4 k+1), 1 /(4 k)]} \cong \bar{\alpha}_{A_{n+k}}$ and $\left.\gamma_{0}\right|_{[1 /(4 k+3), 1 /(4 k+2)]} \cong \alpha_{A_{n+k}}$ for $k \geq 1$

(3) $\left.\gamma_{0}\right|_{[1 / 4 k, 1 /(4 k-1)]}$ is a linear mapping and $\left.\gamma_{0}\right|_{[1 /(4 k+2), 1 /(4 k+1)]}$ is constant for $k \geq 1$.

It is easy to see that $\gamma_{0}$ is homotopic to $\beta$ in $p^{-1}([0,1 / n] \times\{0\})$. This homotopy corresponds to procedure I. Next we describe the homotopy corresponding to procedure II according to the above classification $(1)-(3)$. Let $E_{n, t}$ be the point $((t+n) /((n+1) n) ; t)$ on $L_{2 n}$ and $F_{n, t}$ be the point $(1 / n ; t)$ on $L_{2 n-1}$. We define $H: \mathbb{I} \times \mathbb{I} \rightarrow p^{-1}([0,1 / n] \times \mathbb{I})$ so that $H(s, 0)=\gamma_{0}(s)$ and the following hold:

(a) $H(0, t)=(0 ; t), H(s, t)=(2(1-s) / n ; t)$ for $s \in[1 / 2,1]$ and $\left.H(-, t)\right|_{[1 / 3,1 / 2]} \cong \alpha_{(1 / n ; t)} ;$

(b) $\left.H(-, t)\right|_{[1 /(4 k+1), 1 / 4 k]} \cong \bar{\alpha}_{E_{n+k-1, t}}$ and $\left.H(-, t)\right|_{[1 /(4 k+3), 1 /(4 k+2)]} \cong$ $\alpha_{F_{n+k, t}} ;$

(c) $H(1 /(4 k+2), t)=F_{n+k, t}, H(1 /(4 k+1), t)=H(1 /(4 k), t)=E_{n+k-1, t}$ and $\left.H(-, t)\right|_{[1 / 4 k, 1 /(4 k-1)]}$ and $\left.H(-, t)\right|_{[1 /(4 k+2), 1 /(4 k+1)]}$ are linear mappings.

Then $H$ is continuous and it is a homotopy with $H(0, t)=H(1, t)$ for all $t$. Let $\gamma_{1}=H(-, 1)$. Notice that $\left.\gamma_{1}\right|_{[1 /(4 k+1), 1 /(4 k-2)]} \cong \bar{\alpha}_{B_{n+k-1}} c_{B_{n+k-1}} \alpha_{B_{n+k-1}}$ and $\left.\gamma_{1}\right|_{[1 /(4 k+2), 1 /(4 k+1)]}$ is a linear mapping onto $\left[B_{n+k}, B_{n+k-1}\right]$ and $\left.\gamma_{1}\right|_{[1 / 2,1]}$ is a linear mapping onto $\left[B_{n}, B\right]$.

It is then easy to see that $\gamma_{1}$ is null-homotopic in $p^{-1}([0,1 / n] \times\{1\})$, which corresponds to procedure III.

Proof of Theorem 1.1. By Lemma 3.6, we may start from a loop $f$ : $[0,1] \rightarrow M_{0}$ with base point $A$. Moreover, since the points $A_{m}$ are isolated among $\left\{A_{m}: m<\omega\right\}$, are connected by intervals in $\mathbb{I} \times\{0\}$ and converge to $A$, every loop in $M_{0}$ is homotopic to a loop homotopic to an infinite concatenation of basic loops. We may assume that we have a disjoint family of intervals $\left(a_{n}, b_{n}\right)(n<\nu)$, where $\nu \leq \omega$, such that each $\left.f\right|_{\left[a_{n}, b_{n}\right]}$ is a basic loop at some $A_{m}$ and $\bigcup_{n<\nu}\left(a_{n}, b_{n}\right)$ is dense in $\mathbb{I}$. Then $f(s)=A$ for $s \notin \bigcup_{n<\nu}\left(a_{n}, b_{n}\right)$.

We observe that procedure II in the proof of Lemma 3.7 can be performed uniformly for all $\left.f\right|_{\left[a_{n}, b_{n}\right]}$ 's. Then we obtain a homotopy from $f$ to a loop in $M_{1}$ which consists of possibly infinitely many null-homotopic loops. Since the homotopies which correspond to procedure III converge to $B$, we have a 
homotopy from $f$ to the constant mapping $B$. Extending the domain $[0,1]$ into both directions and adding a path from $A$ to $B$, we have a homotopy from $f$ to the constant mapping $A$ relative to their ends.

4. Proof of Theorem 1.2. We shall show that $S C(Z)$ is noncontractible for every noncontractible space $Z$. Let $S C_{n}(Z)$ be the subspace of $S C(Z)$ defined as $p^{-1}\left(L_{2 n-1} \cup L_{2 n} \cup L_{2 n+1}\right)$.

Definition 4.1. A mapping $f: S C_{n}(Z) \rightarrow S C(Z)$ is said to be flat if $p_{2}\left(f\left(z_{1}\right)\right)=p_{2}\left(f\left(z_{2}\right)\right)$ whenever $p_{2}\left(z_{1}\right)=p_{2}\left(z_{2}\right)$ for $z_{1}, z_{2} \in S C_{n}(Z)$. A homotopy $H: S C_{n}(Z) \times \mathbb{I} \rightarrow S C(Z)$ is said to be flat if the mapping $H(-, t)$ is flat for every $t$.

A similar idea of flatness was used in [6].

Lemma 4.2. Let $n \in \mathbb{N}$ and let $H: S C_{n}(Z) \times \mathbb{I} \rightarrow S C(Z)$ be a mapping such that for every $y \in \mathbb{I}$ and $t \in \mathbb{I}$, the closure of the set $p_{2}\left(H\left(M_{y} \cap\right.\right.$ $\left.\left.S C_{n}(Z), t\right)\right)$ does not contain $\{0,1\}$ and both mappings $H(-, 0)$ and $H(-, 1)$ are flat. Then there exists a flat homotopy from $H(-, 0)$ to $H(-, 1)$.

Proof. Fix the numbers $y$ and $t$. Let $A(y, t)$ and $B(y, t)$ be the infimum and the supremum of the function $p_{2} \circ H(-, t): M_{y} \cap S C_{n}(Z) \rightarrow \mathbb{I}$, respectively. Let

$$
C(y, t)=\frac{A(y, t)}{1+A(y, t)-B(y, t)} .
$$

We note that $A(y, 0)=C(y, 0)=B(y, 0)$ and $A(y, 1)=C(y, 1)=B(y, 1)$, because $H(-, 0)$ and $H(-, 1)$ are flat. Consider the subset $\mathbb{I} \times[A(y, t), B(y, t)]$. Let $\varphi$ be its piecewise linear retraction to the interval $\mathbb{I} \times\{C(y, t)\}$, which is defined by the mappings of vertices (see Figure 1): $\varphi\left(B_{n, 0}\right)=C_{2 n-1}$, $\varphi\left(B_{n, 1}\right)=C_{2 n}, \varphi\left(A_{n+1,0}\right)=C_{2 n}, \varphi\left(A_{n, 1}\right)=C_{2 n-1}$, where $A_{n, 0}$ is the intersection point of $L_{2 n-2}$ and $\{(x ; A(y, t)): x \in \mathbb{I}\}, A_{n, 1}$ is the intersection point of $L_{2 n-1}$ and $\{(x ; A(y, t)): x \in \mathbb{I}\}, B_{n, 0}$ is the intersection point of $L_{2 n-1}$ and $\{(x ; B(y, t))\}$, and $B_{n, 1}$ is the intersection point of $L_{2 n}$ and $\{(x ; B(y, t))\}$.

If $A(y, t)=0$ or $B(y, t)=1$, then $C(y, t)=0$ or $C(y, t)=1$ and $A_{n, 0}=A_{n, 1}=A_{n}$ or $B_{n, 0}=B_{n, 1}=B_{n}$, respectively, and the mapping $\varphi$ is well-defined.

Let $\psi_{y, t}$ be the natural retraction of $p^{-1}(\mathbb{I} \times[A(y, t), B(y, t)])$ to $p^{-1}(\mathbb{I} \times$ $\{C(y, t)\})$ generated by $\varphi$. Define now the homotopy $H^{\prime}: S C_{n}(Z) \times \mathbb{I} \rightarrow$ $S C(Z)$ by $H^{\prime}(z, t)=\psi_{\left(p_{2}(z), t\right)}(H(z, t))$. It is easy to check that $p_{2}\left(H^{\prime}(z, t)\right)=$ $C\left(p_{2}(z), t\right)$ so that $H^{\prime}$ is a flat homotopy, and that $H^{\prime}(-, 0)=H(-, 0)$ and $H^{\prime}(-, 1)=H(-, 1)$.

To prove Lemma 4.4 below we introduce a notion which will help us to investigate flat homotopies.

For $s \in(0,1)$ and $t \in \mathbb{I}$, we define a property $P(s, t)$ of $H$ as follows: 


\begin{abstract}
$H\left(M_{s} \cap S C_{n}(Z), t\right) \subseteq p^{-1}(\mathbb{I} \times(0,1))$ and the restriction of $H(-, t)$ to $M_{s} \cap S C_{n}(Z)$ is homotopic to the identity mapping on $M_{s} \cap S C_{n}(Z)$ in $p^{-1}(\mathbb{I} \times(0,1))$.
\end{abstract}

We remark that by the flatness of $H$, if $H\left(M_{s} \cap S C_{n}(Z), t\right) \subseteq p^{-1}(\mathbb{I} \times(0,1))$, then there is a neighborhood $U$ of $(s ; t)$ such that $H\left(M_{s^{\prime}} \cap S C_{n}(Z), t^{\prime}\right) \subseteq$ $p^{-1}(\mathbb{I} \times(0,1))$ for any $\left(s^{\prime} ; t^{\prime}\right) \in U$.

LEMMA 4.3. Let $Z$ be a noncontractible space and $H: S C_{n}(Z) \times \mathbb{I} \rightarrow$ $S C(Z)$ a flat homotopy. If $p_{2} \circ H\left(M_{0} \cap S C_{n}(Z), t_{0}\right) \subseteq p^{-1}(\mathbb{I} \times(0,1))$, then there exists a neighborhood $U$ of $\left(0, t_{0}\right)$ such that $H$ does not satisfy $P(s, t)$ for any $(s ; t) \in U$ with $s>0$. An analogous statement holds for $p_{2} \circ H\left(M_{1} \cap\right.$ $\left.S C_{n}(Z), t_{0}\right) \subseteq p^{-1}(\mathbb{I} \times(0,1))$.

Proof. We have a neighborhood $U$ of $\left(0, t_{0}\right)$ such that $H\left(M_{s} \cap S C_{n}(Z), t\right)$ $\subseteq p^{-1}(\mathbb{I} \times(0,1))$ for any $(s ; t) \in U$. We fix $(s ; t) \in U$ with $s>0$ and assume to the contrary that $P(s, t)$ holds. Let $P_{2 n+1}, P_{2 n}, P_{2 n-1}$ be the intersections of $\mathbb{I} \times\{s\}$ and $L_{2 n+1}, L_{2 n}, L_{2 n-1}$, respectively, and $I_{2 n}=\left[P_{2 n+1}, P_{2 n}\right]$ and $I_{2 n-1}=\left[P_{2 n}, P_{2 n-1}\right]$. Then we have $M_{s}=\{(0 ; s)\} \cup \bigcup_{n=1}^{\infty} I_{n} \cup$ $Z_{P_{n}}$ and $M_{s} \cap S C_{n}(Z)=Z_{P_{2 n+1}} \cup Z_{P_{2 n}} \cup Z_{P_{2 n-1}}$. Since $H(-, t)$ maps $\bigcup_{u \in\left[P_{2 n+1}, A_{n+1}\right] \cup\left[A_{n+1}, P_{2 n}\right]} Z_{u}$ into $p^{-1}(\mathbb{I} \times(0,1))$, the restriction of $H(-, t)$ to $Z_{P_{2 n+1}} \cup Z_{P_{2 n}}$ is homotopic to a map $f: Z_{P_{2 n+1}} \cup Z_{P_{2 n}} \rightarrow Z_{P_{2 n}}$ in $p^{-1}(\mathbb{I} \times(0,1))$.

Since $M_{s}$ is a strong deformation retract of $p^{-1}(\mathbb{I} \times(0,1))$, as in Lemma 3.5, $Z_{P_{2 n+1}} \cup I_{2 n} \cup Z_{P_{2 n}}$ is a retract of $p^{-1}(\mathbb{I} \times(0,1))$. Since $Z$ is not contractible, the identity mapping on $Z_{P_{2 n+1}} \cup Z_{P_{2 n}}$ is not homotopic to any map $f: Z_{P_{2 n+1}} \cup Z_{P_{2 n}} \rightarrow Z_{P_{2 n}}$ in $p^{-1}(\mathbb{I} \times(0,1))$, which is a contradiction.

To prove the statement for $H\left(M_{1}, t_{0}\right)$ we use $Z_{P_{2 n}} \cup Z_{P_{2 n-1}}$ and argue on a neighborhood of $B_{n}$ to obtain a similar conclusion.

LEMMA 4.4. Let $Z$ be a noncontractible space. If $H: S C_{n}(Z) \times \mathbb{I} \rightarrow$ $S C(Z)$ is a flat homotopy such that $H(u, 0)=u$ for every $u \in S C_{n}(Z)$, then $H(-, 1)$ is not a constant mapping.

Proof. To show this by contradiction, suppose that $H(-, 1)$ is a constant mapping. Let $d:[0,1] \rightarrow S^{1}$ be a winding with base point $s_{0}$, i.e. both $\left.d\right|_{[0,1)}$ and $\left.d\right|_{(0,1]}$ are bijective continuous mappings with $d(0)=d(1)=s_{0}$.

We define a homotopy $H^{*}: S^{1} \times \mathbb{I} \rightarrow S^{1}$ as follows:

$$
H^{*}(u, t)= \begin{cases}d\left(p_{2} \circ H\left(M_{d^{-1}(u)}, t\right)\right) & \text { if } u \neq s_{0} \text { and } P\left(d^{-1}(u), t\right) \text { holds } \\ s_{0} & \text { otherwise. }\end{cases}
$$

If $H^{*}$ is a homotopy, we have a contradiction with the fact that $H^{*}(s, 0)$ $=s$ and that $S^{1}$ is not contractible (cf. [2]). Hence it suffices to verify the continuity of $H^{*}$. 
If $u \neq s_{0}$ and $P\left(d^{-1}(u), t\right)$ holds, the continuity at $(u, t)$ is clear. Otherwise, $u \neq s_{0}$ and $P\left(d^{-1}(u), t\right)$ does not hold, or $u=s_{0}$. We consider two cases:

CASE 1. Suppose that $u \neq s_{0}$ and $P\left(d^{-1}(u), t\right)$ does not hold. If $p_{2} \circ$ $H\left(M_{d^{-1}(u)}, t\right)=\{0\}$ or $\{1\}$, then the continuity at $(u, t)$ follows from that of $H$. Otherwise, since $H(-, t)$ maps $M_{d^{-1}(u)} \cap S C_{n}(Z)$ continuously with respect to $u$ and $t$, the restriction of $H(-, t)$ to $M_{d^{-1}(u)} \cap S C_{n}(Z)$ is not homotopic to the identity on $M_{d^{-1}(u)} \cap S C_{n}(Z)$ in $p^{-1}(\mathbb{I} \times(0,1))$, i.e. $H^{*}$ takes the value $s_{0}$ in a neighborhood of $(u, t)$.

CASE 2. Suppose that $u=s_{0}$. If each of $p_{2} \circ H\left(M_{0} \cap S C_{n}(Z), t\right)$ and $p_{2} \circ H\left(M_{1} \cap S C_{n}(Z), t\right)$ is equal to either $\{0\}$ or $\{1\}$, the continuity at $(u, t)$ follows from that of $H$. The remaining case is when $p_{2} \circ H\left(M_{0} \cap S C_{n}(Z), t\right) \subseteq$ $(0,1)$ or $p_{2} \circ H\left(M_{1} \cap S C_{n}(Z), t\right) \subseteq(0,1)$. In this case the continuity follows by Lemma 4.3 .

Proof of Theorem 1.2. To get a contradiction, suppose that $S C(Z)$ is a contractible space. Then there exists a contraction $H: S C(Z) \times \mathbb{I} \rightarrow S C(Z)$. By the compactness of the time interval $\mathbb{I}$, for every $a=(0 ; y) \in\{0\} \times \mathbb{I}$, there exists $\varepsilon_{0}>0$ such that the diameters of $p_{2} \circ H\left(O_{\varepsilon_{0}}(a), t\right)$ are less than 1 for all $t \in \mathbb{I}$. Hence, by compactness of $L$, there exists $\varepsilon_{1}>0$ such that the diameters $p_{2} \circ H\left(O_{\varepsilon_{1}}(a), t\right)$ are less than 1 for all $a=(0 ; y) \in\{0\} \times \mathbb{I}$ and all $t \in \mathbb{I}$. Let $n$ be a number such that $1 / n<\varepsilon_{1}$. By Lemma 4.2 we may assume that $\left.H\right|_{S C_{n}(Z) \times \mathbb{I}}$ is a flat contraction. However, this contradicts Lemma 4.4.

REMARK 4.5. The space $S C\left(S^{1}\right)$ is simply connected and it follows by the Mayer-Vietoris exact sequence for singular homology that $H_{n}\left(S C\left(S^{1}\right)\right)$ $=0$ for $n \geq 3$. The question whether $H_{2}\left(S C\left(S^{1}\right)\right)=0$ was open in the first draft of this paper. This has subsequently been answered negatively by the authors and also independently by J. Dydak and A. Mitra. The proof will appear in our forthcoming paper "An example of a nonaspherical cell-like 2-dimensional continuum and some related constructions".

Acknowledgements. The authors thank the referee for careful reading, detecting errors, and suggestions to make arguments clearer. They also thank Haruto Ohta for discussions about dimension theory. The first and third authors were supported by the Japanese-Slovenian research grant BI-JP/0304/2 and the Grant-in-Aid for Scientific Research (C) of Japan No. 16540125.

\section{References}

[1] K. Borsuk, Theory of Retracts, PWN, Warszawa, 1967.

[2] W. Dębski, Pseudo-contractibility of the $\sin (1 / x)$-curve, Houston J. Math. 20 (1994), 365-367. 
[3] K. Eda and K. Kawamura, Homotopy and homology groups of the n-dimensional Hawaiian earring, Fund. Math. 165 (2000), 17-28.

[4] R. Engelking, Theory of Dimensions, Finite and Infinite, Heldermann, Lemgo, 1995.

[5] H. B. Griffiths, The fundamental group of two spaces with a common point, Quart. J. Math. Oxford (2) 5 (1954), 175-190; Correction, ibid. 6 (1955), 154-155.

[6] U. H. Karimov and D. Repovš, On contractible polyhedra that are not simply contractible, Proc. Amer. Math. Soc. 7 (2004), 2159-2162.

[7] R. C. Lacher, Cell-like mappings and their generalizations, Bull. Amer. Math. Soc. 83 (1977), 495-552.

[8] W. J. R. Mitchell and D. Repovš, Topology of cell-like mappings, in: Proc. Conf. Diff. Geom. and Topol. (Cala Gonone, 1988), Suppl. Rend. Fac. Sci. Nat. Univ. Cagliari 58 (1988), 265-300.

[9] K. Morita, On the dimension of product spaces, Amer. J. Math. 75 (1953), 205-223.

[10] E. H. Spanier, Algebraic Topology, McGraw-Hill, New York, 1966.

School of Science and Engineering

Waseda University

Tokyo 169-8555, Japan

E-mail: eda@logic.info.waseda.ac.jp

Institute of Mathematics, Physics and Mechanics

University of Ljubljana

P.O. Box 2964

Ljubljana 1001, Slovenia

E-mail: dusan.repovs@guest.arnes.si
Institute of Mathematics Academy of Sciences of Tajikistan Ul. Ainy $299^{\mathrm{A}}$ Dushanbe 734063, Tajikistan E-mail: umed-karimov@mail.ru

Received 4 January 2005;

in revised form 5 December 2006 